\title{
Optimal turbine spacing in fully developed wind-farm boundary layers
}

\author{
Johan Meyers $^{1}$ and Charles Meneveau ${ }^{2}$ \\ ${ }^{1}$ Department of Mechanical Engineering, Katholieke Universiteit Leuven, \\ Celestijnenlaan 300A — bus 2421, B3001 Leuven, Belgium \\ ${ }^{2}$ Department of Mechanical Engineering \& Center for Environmental and \\ Applied Fluid Mechanics, Johns Hopkins University, \\ 3400 North Charles Street, Baltimore MD 21218, USA
}

February 11, 2011

\begin{abstract}
As wind farms become larger, the asymptotic limit of the "fully developed", or "infinite", wind farm has been receiving increased interest. This limit is relevant for wind farms on flat terrain whose length exceeds the height of the atmospheric boundary layer by over an order of magnitude. Recent computational studies based on Large Eddy Simulation have identified various mean velocity equilibrium layers, and have led to parameterizations of the effective roughness height that allow predicting the wind velocity at hub-height as function of parameters such as wind turbine spacing and loading factors. In the current paper, we employ this as a tool to make predictions of optimal wind turbine spacing as function of these parameters, as well as in terms of the ratio of turbine costs to land-surface costs. For realistic cost ratios, we find that the optimal average turbine spacing may be considerably higher than conventionally used in current wind-farm implementations.
\end{abstract}

Keywords: wind farm, wind energy, optimal wind turbine spacing, large-eddy simulation

\section{INTRODUCTION}

Recently, wind energy has received renewed interest. This originates in part from large funding programs by American and European governments, and comes from the realization that wind energy will be an important contributor in the production of affordable and clean energy in the next decades. In various scenarios, ${ }^{1,2}$ a contribution of wind energy to the overall electricity production up to $20 \%$ is aimed at by 2030. To realize these targets, larger wind farms (both on- and off-shore), covering increasingly larger surface areas are required. When large-scale wind-farm implementations are considered, the total drag induced by all turbines in the farm may change the equilibrium in the atmospheric surface layer. In particular, with a characteristic height of the ABL of about $1 \mathrm{~km}$, wind farms with horizontal extents exceeding 10-20 km may therefore approach the asymptotic limit of "infinite" wind farms, and the boundary layer flow may approach a new fully developed regime, which depends on the additional surface drag induced by the wind farm. In the current study, we focus 
on this asymptotic "infinite" wind-farm regime, and investigate the optimal wind-turbine spacing in these wind farms to either optimize the ratio of total power output per land surface, or the ratio of total power output per unit of total cost that also includes cost of turbines. Depending on the ratio between total costs per turbine and total costs per land surface, in the case of "infinite" wind farms, we find that the optimal average turbine spacing may be considerably higher then conventionally used in current wind-farm implementations.

Design and optimization of single wind turbines is well explored nowadays, often using bladeelement-momentum theory, and Glauert's theory for rotor aerodynamics. ${ }^{3,4}$ Also effects of turbine wake aerodynamics have received much attention. ${ }^{5}$ Studies of the interaction of large wind farms and the atmospheric boundary layer (ABL) are far less prevalent. In this area, pioneering work was performed by Frandsen, ${ }^{6}$ who formulated a model for the surface roughness induced by "infinite" wind turbine arrays. More recently, the subject gained renewed interest in the context of off-shore windfarm under performance. ${ }^{7}$ Very recently, studies employed large-eddy simulations to study windfarm-ABL interactions, ${ }^{8,9}$ focusing on the 'infinite' wind-farm limit. Moreover, in Ref. 8, Frandsen's model for the induced wind-farm surface roughness was refined, to include effects of turbine-wake mixing.

When turbine spacing is considered in a more conventional approach, minimum wind-turbine spacing in wind farms is mainly governed by the desire to limit wake-induced fatigue loads in turbines located downstream of a prior row of turbines. ${ }^{5}$ However, large wind farms increase the effective surface roughness experienced by the $\mathrm{ABL},{ }^{6,8}$ such that the effective wind velocity at turbine-hub height decreases compared to an unloaded ABL. Hence, increasing the installed power per land surface area (i.e. decreasing the average wind-turbine spacing) has an inverse effect on the total extracted power per turbine. Depending on the cost per turbine, and the cost of land used for wind farms, this leads to an optimization problem for wind-turbine spacing in wind farms, where the optimal spacing is given by economical constraints. In the current work the refined effective roughness model of Ref. 8 is used as the basis to elaborate a model for overall wind-farm power output per land surface, taking fully developed wind-farm-ABL interactions into account. A detailed discussion is presented on optimal turbine spacing, and its dependence on economical parameter, and operating regimes.

Wind-turbine operation is often classified into three regions: region I-III. ${ }^{4,10}$ The first region is at very low wind speeds where aerodynamic forces cannot overcome the turbine's internal friction losses. At very high wind speeds (Region III), the power output of turbines is restricted by loading constraints on its mechanical structures and by economical constraints on the size of the power generator. In this region, turbine power is controlled at a constant level, independent of wind speed, either by stalling the turbine blades, or by feathering the turbine. ${ }^{4}$ In region II, power output is not restricted, and wind turbines work close to their aerodynamical optimal operating conditions. In the current work, we focus in large part on optimization of turbine-spacing in region II, where turbine thrust and power coefficients are close to optimal. At the end of Section 3, region III operation and its influence on optimal turbine spacing in wind farms, is discussed. It will be argued that feathering may have an impact on the optimal turbine spacing in the equilibrium wind-farm ABL, while stalling the turbine keeps the optimal spacing at the region-II optimum.

The paper is further organized as follows. First, in Section 2, the model for wind-farm optimization is elaborated. In Section 3, optimization results are presented and discussed. Finally, conclusions are presented in Section 4.

\section{MODEL FOR WIND-FARM OPTIMIZATION}

First, some definitions and conventions for wind-turbine thrust and power, which will be further used in the current study, are introduced in $\$ 2.1$. Next, in $\$ 2.2$, standard relations for the atmospheric 
boundary layer are briefly reviewed. Subsequently, the induced surface-roughness model for wind farms ${ }^{8}$ is discussed in $\S 2.3$. Finally, in $\S 2.4$ the wind-farm optimization problem is defined in terms of normalized farm power.

\subsection{Definitions and conventions}

In conventional wind-turbine momentum theory, the thrust of a single wind-turbine on the surrounding flow is expressed as

$$
F_{T}=-\frac{1}{2} C_{T} \rho U_{\infty}^{2} A,
$$

with $C_{T}$ the thrust coefficient, $U_{\infty}$ the upstream undisturbed flow velocity at hub height, and $A=$ $\pi D^{2} / 4$ the turbine-rotor area (with $D$ the rotor diameter). However, for large wind-turbine arrays with significant interactions among wind turbines and wakes, this reference velocity $U_{\infty}$ is not readily known and would require arbitrary decisions about what upstream distance to use when specifying the velocity. Moreover, such a reference velocity would depend on farm parameters such as the average turbine spacing, and turbine loading. Instead, for wind farms, it is useful to base the relations for thrust on the prevailing axial velocity at the rotor-disk position, $U_{d}$, such that

$$
F_{T}=-\frac{1}{2} C_{T}^{\prime} \rho U_{d}^{2} A
$$

Note that the value of $C_{T}^{\prime}$ is straightforwardly related to the lift and drag coefficients of the turbine blades (see e.g. Ref. 9 for an elaboration), and much less sensitive to farm parameters such as average turbine spacing. Moreover, in large-eddy simulations of wind farms, ${ }^{8} U_{d}$ is readily available during the simulation, such that Eq. (2) can be directly employed as a force model.

For a lone-standing turbine, it is possible to relate $C_{T}^{\prime}$ to $C_{T}$ by using classic actuator disk theory. This allows us to express

$$
U_{d}=U_{\infty}(1-a), \quad C_{T}^{\prime}=\frac{C_{T}}{(1-a)^{2}},
$$

with $a$ the axial induction factor. ${ }^{9}$ For the Betz limit ${ }^{4}$ (i.e., $C_{T}=8 / 9$, and $a=1 / 3$ ), we obtain $C_{T}^{\prime}=2$. Using typical values $C_{T}=0.75$, and $a=1 / 4$ (which have been used before for modeling wind turbines $)^{11}$ leads to $C_{T}^{\prime}=4 / 3$. Obviously, for wind farms, Eq. (3) is not valid, though the typical values for $C_{T}^{\prime}$ remain applicable.

For wind-turbine farms, it is further useful to express the thrust in relation to the average land surface area $S$ per turbine $\left(S=\ell_{x} \ell_{y}\right.$, with $\ell_{x}, \ell_{y}$ the average turbine-spacing in stream-wise and span-wise directions), leading to

$$
F_{T}=\frac{1}{2} c_{f t}^{\prime} \rho U_{d}^{2} S,
$$

with a friction coefficient $c_{f t}^{\prime}$ based on the horizontal surface rather than frontal area. Further,

$$
c_{f t}^{\prime}=\frac{\pi C_{T}^{\prime}}{4 s_{x} s_{y}}=\frac{\pi C_{T}^{\prime}}{4 s^{2}}
$$

with $s_{x}=\ell_{x} / D, s_{y}=\ell_{y} / D$, and $s=\sqrt{s_{x} s_{y}}$.

The power extracted on average by wind turbines from the atmospheric boundary layer corresponds to

$$
P=\frac{1}{2} C_{T}^{\prime} \rho U_{d}^{3} A=\frac{1}{2} c_{f t}^{\prime} \rho U_{d}^{3} S .
$$

This is not equivalent to the power $P_{a x}$ on the turbine axis. The latter relates to the torque and rotational velocity of the turbine. The drag forces on the turbine blades increase thrust, but reduce torque. From 
an energetic point of view, the drag forces lead to losses, corresponding to a conversion of mean-flow energy in the atmospheric boundary layer into turbulent motion and heat. Using the power coefficient $C_{P}$, and $C_{P}^{\prime}$ (respectively with respect to $U_{\infty}$, and $U_{d}$ ), the power on the turbine axis corresponds to

$$
P_{a x}=P \frac{C_{P}^{\prime}}{C_{T}^{\prime}}=P \frac{C_{P}}{C_{T}(1-a)}
$$

Using actuator disk theory, it is straightforward to find that $C_{P}=C_{P}^{\prime}(1-a)^{3}$. For the Betz limit (i.e., $C_{P}=16 / 27$, and $\left.a=1 / 3\right), C_{P}^{\prime}=2.0$.

For wind turbines, typical optimal values may be $C_{P} \approx 0.34$ and $a \approx 1 / 4$, such that $C_{P}^{\prime} \approx 0.8$, and $P_{a x} \approx 0.6 P$. In reality, the ratio $C_{P}^{\prime} / C_{T}^{\prime}$ depends on the turbine working region. In region II, $C_{P}^{\prime} / C_{T}^{\prime}$ is close to optimal, with high values for $C_{T}^{\prime}$, and $C_{P}^{\prime}$. Consequently, in this operating region, optimization to $P$ or $P_{a x}$ is roughly equivalent. In region III, the turbine's power output is controlled to be constant. Depending on the control mechanism, this may lead to a large decrease in $C_{P}^{\prime} / C_{T}^{\prime}$. Consequences of region III operation on the optimization results in the current work are addressed, separately, at the end of Section 3. Until then, we assume $P \sim P_{a x}$, and formulate the wind-farm-ABL optimization problem in terms of $P$.

\subsection{Geostrophic wind and $A B L$ relations}

In the current subsection, we briefly review classical relations for the atmospheric boundary layer, as, e.g., well documented in Ref. 12.

In the atmospheric boundary layer (ABL), the driving force is the geostrophic wind, of velocity magnitude $G$, on top of the ABL, which is given by geostrophic balance condition without the effects of friction. Inside the boundary layer, a balance exists between pressure forces, Reynolds stresses, and Coriolis forces induced by the Earth's rotation. Since the velocity in the ABL decreases towards the surface, Coriolis forces also decrease, which causes the velocity to turn away from the geostrophic wind direction at lower altitudes, often referred to as the Ekman spiral. Conventionally, a reference frame is selected which is aligned with the wind speed near the surface (in the inner layer of the boundary layer). In this case, the geostrophic wind $G$ is defined with two components, i.e. $U_{G}$ in stream-wise, and $V_{G}$ in span-wise direction, such that $G=\left(U_{G}^{2}+V_{G}^{2}\right)^{1 / 2}$, and $\gamma=\arctan \left(-V_{G} / U_{G}\right)$ the angle between the geostrophic wind direction, and the wind direction near the surface. Classical similarity theory then leads to ${ }^{12}$

$$
\begin{aligned}
\frac{U_{G}}{u_{*}} & =\frac{1}{\kappa} \ln \left(\frac{u_{*}}{f z_{0}}\right)-C, \\
\frac{V_{G}}{u_{*}} & =-A
\end{aligned}
$$

with $\kappa=0.4$ the Von Kármán constant, and where $C \approx 4.5$, and $A \approx 11.25$ are found to be good values. ${ }^{6}$ Further, $z_{0}$ is the surface roughness. In the context of wind-farms, this is related to total roughness induced by the ground surface and the wind turbines on the ABL. Likewise, $u_{*}$ is the friction velocity, which is related to the total friction exerted by the ground and wind turbines on the boundary layer. Further details on $z_{0}$, and $u_{*}$, and their relation to the wind-farm parametrization, etc., are provided in $\S 2.3$. Finally,

$$
f=2 \Omega \sin \phi
$$

is the Coriolis parameter. For $\Omega=2 \pi /(24 \times 3600 s)=7.27 \times 10^{-5} 1 / \mathrm{s}$, and, e.g., at 40 degree latitude, we get $f=9.34 \times 10^{-5} 1 / \mathrm{s}$. 


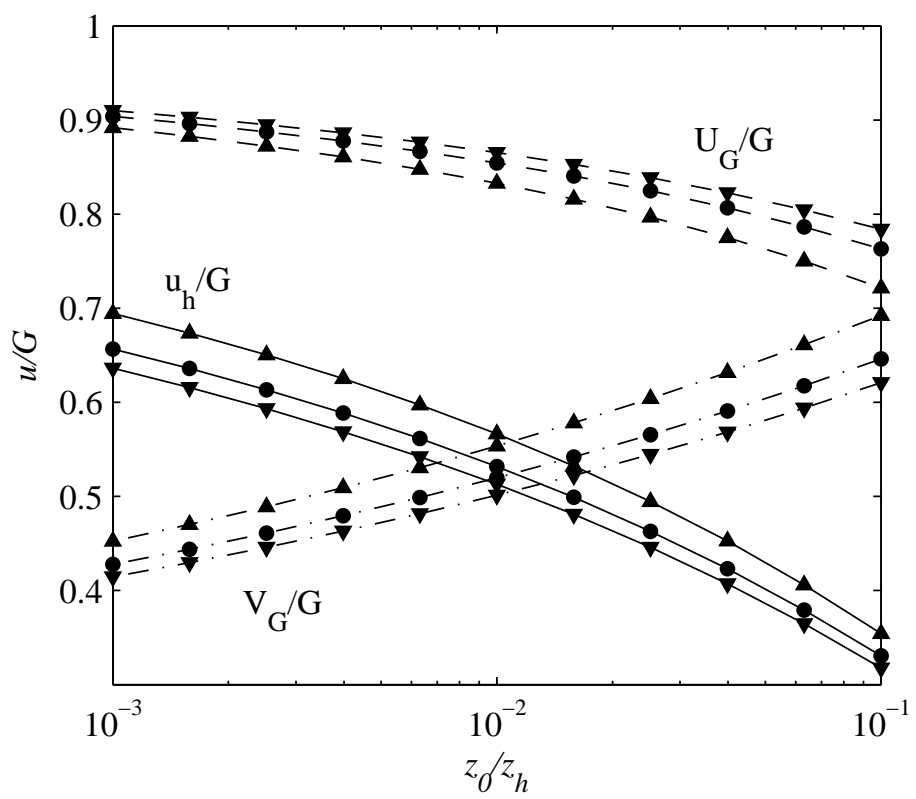

Figure 1: Relation between geostrophic wind and wind speed at turbine hub height as function of the surface roughness in the ABL (cf. Eqs. $(8,9,11)$ with $f=9.34 \times 10^{-5} 1 / \mathrm{s}$, and $\left.z_{h}=100 \mathrm{~m}\right) .(-): u_{h} / G$; $(--): U_{G} / G$; and (-.): $V_{G} / G .(\Delta): R o_{h}=1000 ;(\bullet): R o_{h}=2000 ;$ and $(\mathbf{\nabla}): R o_{h}=3000$;

Combining Eq. (8), and (9), leads to

$$
\frac{G}{u_{*}}=\sqrt{A^{2}+\left[\frac{1}{\kappa} \ln \left(\frac{u_{*}}{G} R o\right)-C\right]^{2}},
$$

where the dimensionless group $R o=G /\left(f z_{0}\right)$ has the form of a Rossby number, expressing a ratio between inertia and Coriolis forces. In the current work, we are mainly interested in the reaction of the ABL to changes in the surface roughness induced by wind turbines. Therefore, we introduce an alternative Rossby number, using the turbine hub-height as reference length scale, such that

$$
R o_{h}=\frac{G}{f z_{h}}=R o \frac{z_{0}}{z_{h}},
$$

and we will evaluate the effect of variations in $z_{0} / z_{h}$, while keeping $R o_{h}$ constant. A representative reference value for $R o_{h}$ may, e.g., be estimated using $f=9.34 \times 10^{-5} 1 / \mathrm{s}, G=20 \mathrm{~m} / \mathrm{s}$, and $z_{h}=100 \mathrm{~m}$, leading to $R o_{h} \approx 2140$.

Using expression (11), it is useful to investigate the relation between the geostrophic velocity $G$, and $u_{h}$, the mean streamwise velocity at turbine hub-height, which we estimate here using MoninObukhov similarity under neutral stratification conditions (the log-law for rough walls). One can write $u_{h} \approx u^{*} / \kappa \ln \left(z_{h} / z_{0}\right)$, with $z_{h}$ the turbine-hub height. To this end, $G / u_{*}$ is solved numerically from Eq. (11), using MATLAB's fsolve function. Alternatively, fits to the inverse function may be employed, as, e.g., proposed in Ref. 7 and further explored in the Appendix, where such an approximate expression is given explicitly (since it involves errors on the order of $7 \%$ for $G / u_{*}$, here we continue to use the numerical solution). In Figure $1, u_{h} / G$ is displayed, together with the separate geostrophic components $U_{G} / G$, and $V_{G} / G$ as function of the surface roughness $z_{0}$ (with $z_{0}$ covering a range between $0.1 \mathrm{~m}$ and $10 \mathrm{~m}$ - as may be encountered in large wind farms ${ }^{8}-$ normalized by $z_{h}=100$ ). In the figure, three different values of $R o_{h}$ are displayed, i.e. $R o_{h}=1000, R o_{h}=2000$, and $R o_{h}=3000$. 
It is appreciated that $u_{h} / G$ drops significantly when the surface roughness $z_{0}$ increases, reducing the available wind speed at hub height. Figure 1 also illustrates that the angle between the geostrophic wind direction, and the surface wind direction increases when $z_{0}$ increases, as is apparent from the changes in $U_{G} / G$, and $V_{G} / G$. The induced roughness $z_{0}$ in a wind farms strongly depends on the average turbine spacing $s$, and the thrust coefficient $C_{T}^{\prime}$. Hence, since the geostrophic wind $G$ is the driving force in the ABL, the strong dependence of $u_{h} / G$ on $z_{0}$ should be taken into account when wind-farm lay-out for optimal power output is considered. This is addressed in the next section. For this analysis to follow, we will keep the Rossby number $R o_{h}$ constant at 2000 .

\subsection{Wind-farm induced surface roughness from LES}

Depending on atmospheric conditions, the magnitude of the geostrophic wind, and surface roughness, the height $H$ of the atmospheric boundary layer typically is of the order of $1-2 \mathrm{~km}$. Consequently, wind turbines, with a typical hub height of $100 \mathrm{~m}$ are situated within the ABL's inner region $(\leq 0.1 H)$. In the classical view on boundary layers, 'outer-layer' and 'inner-layer' dynamics are presumed to be independent (see, e.g., Ref. 13), and the inner layer dynamics are characterized by the surface roughness $z_{0}$, and the friction velocity (characterizing the overall wall friction). In the context of wind farms, Fransden ${ }^{6}$ formulated a model for the surface roughness induced by the farm.

Based on a suite of large-eddy simulation cases, this model was recently refined, including effects of turbine-wake mixing in the formulation. ${ }^{8}$ Specifically, the simulations used periodic boundary conditions in horizontal planes to represent fully developed conditions relevant to wind farms that are 10-20 times longer than the ABL height. For illustration, figure 2 shows contours of streamwise velocity on three perpendicular planes across a snapshot of the flow. Domains containing, e.g. $6 \times 8$ wind turbines were used. The wind turbines were represented using the 'drag-disk' model. In a recent detailed validation study ${ }^{14}$ it was demonstrated that, except for near-wake effects close to the turbines with $x \leq 3 D$, these models allow an accurate representation of the overall wake structures behind turbines. Moreover, also Reynolds stresses, which are responsible for the main vertical fluxes of energy towards the wind turbines, ${ }^{8,15}$ were found to be accurately predicted, ${ }^{14}$ thus allowing an accurate representation of the interaction of the wind farms with the atmospheric boundary layer.

The flow was forced using a streamwise pressure gradient (instead of Coriolis forcing) with the understanding that the 'outer forcing' method should not significantly affect the 'inner-layer' structure of the flow, i.e. the relationship between the resulting roughness and, e.g. the hub-height $z_{h}$. As can be seen in Fig. 2, the wakes meander back and forth before interacting with the next wind turbine row. Also, in the vertical direction, large-scale structures mix the fluid momentum thus entraining kinetic energy into the region where the wind turbines are located. As discussed in detail in Refs. 8, 15, such vertical entrainment is a crucial mechanism in the limit of infinite wind farms.

The suite of LES were processed to obtain horizontally averaged streamwise velocity profiles. As a confirmation of an important assumption made in the original Frandsen $(1992)^{6}$ model, the simulations showed that in the inner layer of a fully developed wind-turbine atmospheric boundary layer, two equilibrium ( $\log$ ) layers exist. The first equilibrium layer, the 'high' layer, is situated above the wind turbine canopy, with a friction velocity $u_{* h i}$ ("high" denoted by subscript ' $h i$ '). This friction velocity is associated with the total friction induced by the ground surface and the wind turbines, balancing the driving forces in the ABL. At very high Reynolds number, it can also be expected that $u_{* h i} \approx\left(-\left\langle\overline{u^{\prime} w^{\prime}}\right\rangle_{z}\right)^{1 / 2}$ (with $-\left\langle\overline{u^{\prime} w^{\prime}}\right\rangle_{z}$ the Reynolds stresses at a height $z$, with $H \gg z \gtrsim z_{h}$ ). A second "low" layer exists below the wind turbine array ("low" denoted by subscript ' $l o$ '), where the friction velocity is reduced due to the momentum lost to the wind-turbines, and equals $u_{* l o}=\sqrt{\tau_{w} / \rho}$, where $\tau_{w}$ is the stress at the ground. A new observation was made based on the LES results, namely of a third layer separating the two log-layers, namely a wake-mixing region at turbine hub height. ${ }^{8}$ 


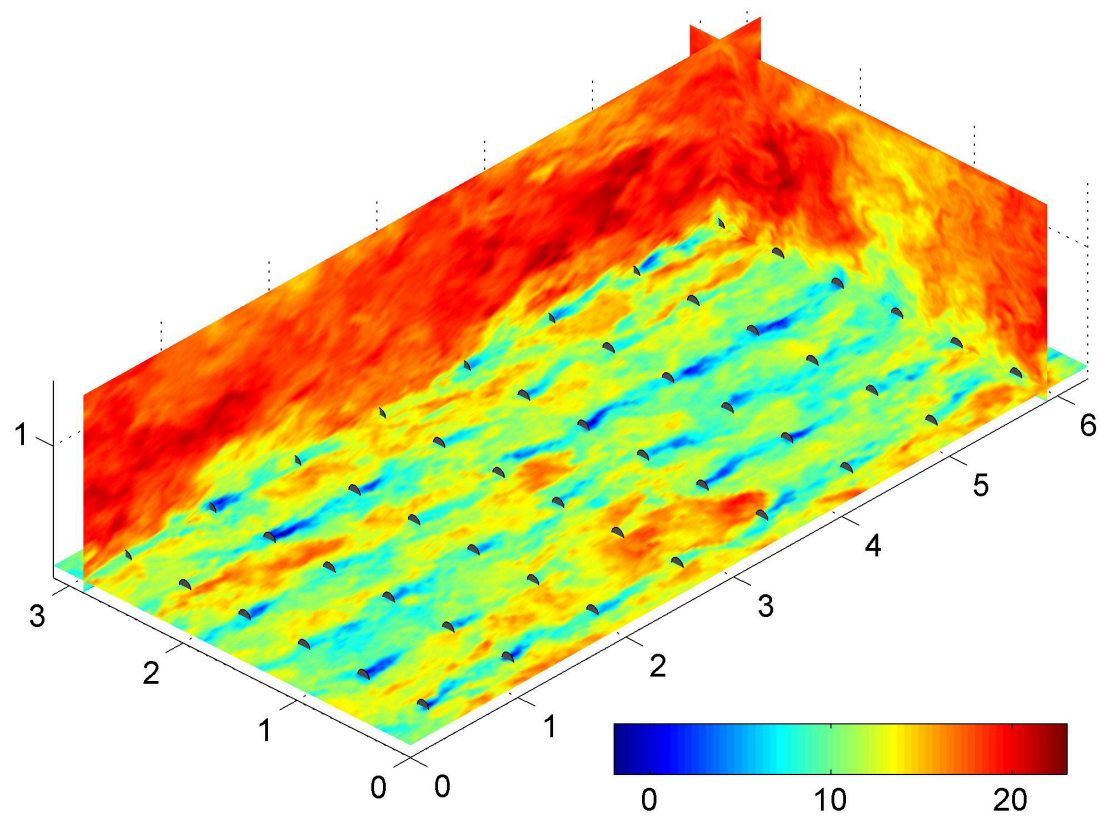

Figure 2: Contours of streamwise velocity (in units of $u_{* h i}$ ) on three orthogonal planes across the domain, obtained from LES. ${ }^{8}$ The horizontal plane cuts the wind turbine rotor planes at hub-height. The model for effective roughness height associated to large wind farms was developed using a suite of such LES under various geometric and loading conditions. ${ }^{8}$

Using the insights from the LES, in Ref. 8, a new model was proposed for the ratio $u_{* l o} / u_{* h i}$, by modifying Frandsen's original model ${ }^{6}$ to include effects of the wake making in the third layer. The ratio between the friction velocities corresponds to ${ }^{8}$

$$
\frac{u_{* l o}}{u_{* h i}}=\frac{\ln \left[\frac{z_{h}}{z_{0, h i}}\left(1+\frac{D}{2 z_{h}}\right)^{\frac{v_{w}^{*}}{1+v_{w}^{*}}}\right]}{\ln \left[\frac{z_{h}}{z_{0, l o}}\left(1-\frac{D}{2 z_{h}}\right)^{\frac{v_{w}^{*}}{1+v_{w}^{*}}}\right]},
$$

where $z_{0, h i}$ is the surface roughness induced by the wind farm, and $v_{w}^{*}$ is a normalized "augmented wake eddy viscosity", estimated as ${ }^{8}$

$$
v_{w}^{*} \approx 28 \sqrt{c_{f t} / 2}
$$

with $c_{f t}=\pi C_{T} / 4 s^{2}$. This normalized eddy viscosity corresponds to the extra eddy viscosity introduced by the turbine wakes in the ABL, normalized with the boundary layer eddy-viscosity $\kappa u_{*} z$, in absence of turbines. ${ }^{8}$ In the surface-roughness model introduced in Ref. 8, it follows from an eddy viscosity formulation which is used to estimate the logarithmic slope of the mean velocity profile at turbine hub height, connecting the "low" and "high" equilibrium layers. Typical values for $v_{w}^{*}$ obtained from large-eddy simulations in Ref. 8 range from 0.5 to 3.5 .

Similar to the friction velocities, a high surface roughness $z_{0, h i}$, and a low surface roughness $z_{0, l o}$ are respectively associated to the upper and lower equilibrium layer. The 'low' surface roughness $z_{0, l o}$ is the standard roughness associated with the land surface length-scales on which the wind farm is build. The high surface roughness, is the surface roughness felt by the equilibrium layer above the 
turbines. In the new model, it is given by ${ }^{8}$

$$
\frac{z_{0, h i}}{z_{h}}=\left(1+\frac{D}{2 z_{h}}\right)^{\frac{v_{w}^{*}}{1+v_{w}^{*}}} \exp \left(-\left[\frac{c_{f t}}{2 \kappa^{2}}+\left(\ln \left[\frac{z_{h}}{z_{0, l o}}\left(1-\frac{D}{2 z_{h}}\right)^{\frac{v_{w}^{*}}{1+v_{w}^{*}}}\right]\right)^{-2}\right]^{-1 / 2}\right)
$$

Using the wind-farm induced surface roughness $z_{0, h i}$, and the relations presented in $\$ 2.2$ (and replacing $z_{0}$ with $z_{0, h i}$ ) we are able to evaluate the effect of wind farms on the ABL. To this end, we rely in the current study on formulations using $c_{f t}^{\prime}$ (cf. \$2.1, and next subsection), while in the model proposed by Calaf et al., $c_{f t}$ is used. In the context of wind farms, the relation between both is not necessarily straightforward, as discussed in $\$ 2.1$. Here, we will use the strong approximation that $c_{f t} \approx 9 / 16 c_{f t}^{\prime}$, i.e. using Eq. (3), with $a=1 / 4$.

In terms of the geostrophic wind and ABL relations introduced in $\S 2.2$, it is $z_{0, h i}$, and $u_{* h i}$, which are important. These are respectively the roughness height and friction velocity experienced by the ABL above the wind farm. Hence, $u_{*}$, and $z_{0}$ in Eqs. (8)-(9), and (11)-(12) should be replaced by $z_{0, h i}$, and $u_{* h i}$ in the context of wind farms.

\subsection{Normalized farm power and optimization problem}

We now turn to the optimization problem. We focus on the normalized farm power, which serves as a basis for the definition of the cost function in the optimization. The power output normalized using the geostrophic wind $G$, and per unit land surface corresponds to

$$
P^{+}\left(s, C_{T}^{\prime}\right)=\frac{P}{S \rho G^{3} / 2}=\frac{\frac{1}{2} C_{T}^{\prime} \rho U_{d}^{3} A}{S \rho G^{3} / 2}=\frac{\pi C_{T}^{\prime}}{4 s^{2}}\left(\frac{u_{* h i}}{G}\right)^{3}\left(\frac{U_{d}}{u_{* h i}}\right)^{3}=c_{f t}^{\prime}\left(\frac{u_{* h i}}{G}\right)^{3}\left(\frac{U_{d}}{u_{* h i}}\right)^{3}
$$

where $G / u_{* h i}$ is given by Eq. (11), and an expression for the ratio of turbine disk velocity to friction velocity, $U_{d} / u_{* h i}$, will be further addressed below.

When optimizing wind-farm power output, it may be relevant to normalize power with total cost instead of total surface area per turbine in the farm. The total cost can be divided into two parts. A first part, consists of costs which are proportionally related to the area of land used, which we will denote here with $\operatorname{cost}_{L}$, expressed in units of $\$ / \mathrm{m}^{2}$. Elements contributing, may be the lease price of land, cost of connectivity to the power net, electric lines and civil works (e.g. in off-shore farms this is a large cost), ${ }^{16}$ etc. A second part of the total cost, consists of costs which are proportional to the number of turbines employed, and we denote the cost per turbine with $\operatorname{cost}_{T}$ [\$]. The ratio of both costs is now defined as

$$
\alpha=\frac{\operatorname{cost}_{T} / A}{\operatorname{cost}_{L}},
$$

where the turbine-rotor-disk area $A$ is used to ensure that $\alpha$ is a non-dimensional constant.

Using these elements, the normalized power per unit cost is now straightforwardly defined as

$$
P^{*}\left(s, C_{T}^{\prime}, \alpha\right)=P^{+} \frac{\operatorname{cost}_{L}}{\operatorname{cost}_{T} / S+\operatorname{cost}_{L}}=P^{+} \frac{4 s^{2} / \pi}{\alpha+4 s^{2} / \pi}=\frac{C_{T}^{\prime}}{\alpha+4 s^{2} / \pi}\left(\frac{u_{* h i}}{G}\right)^{3}\left(\frac{U_{d}}{u_{* h i}}\right)^{3},
$$

where in a first step $\operatorname{cost}_{T}$ is related to the surface area per turbine $S$ to allow a dimensional meaningful addition of costs, and the ratio $\alpha$ is subsequently introduced by rearranging the equation. It is obvious that, by construction, $P^{*}\left(s, C_{T}^{\prime}, 0\right)=P^{+}\left(s, C_{T}^{\prime}\right)$.

To proceed, we will eliminate $U_{d} / u_{* h i}$ from the equations by using a stream-wise momentum balance of the ABL, horizontally averaged per turbine, and integrated over its full height. The balance corresponds to

$$
\int_{0}^{H} f \rho\left(V(z)-V_{G}\right) \ell_{x} \ell_{y} \mathrm{~d} z=\tau_{w} \ell_{x} \ell_{y}+\frac{1}{2} c_{f t}^{\prime} \rho U_{d}^{3} S
$$




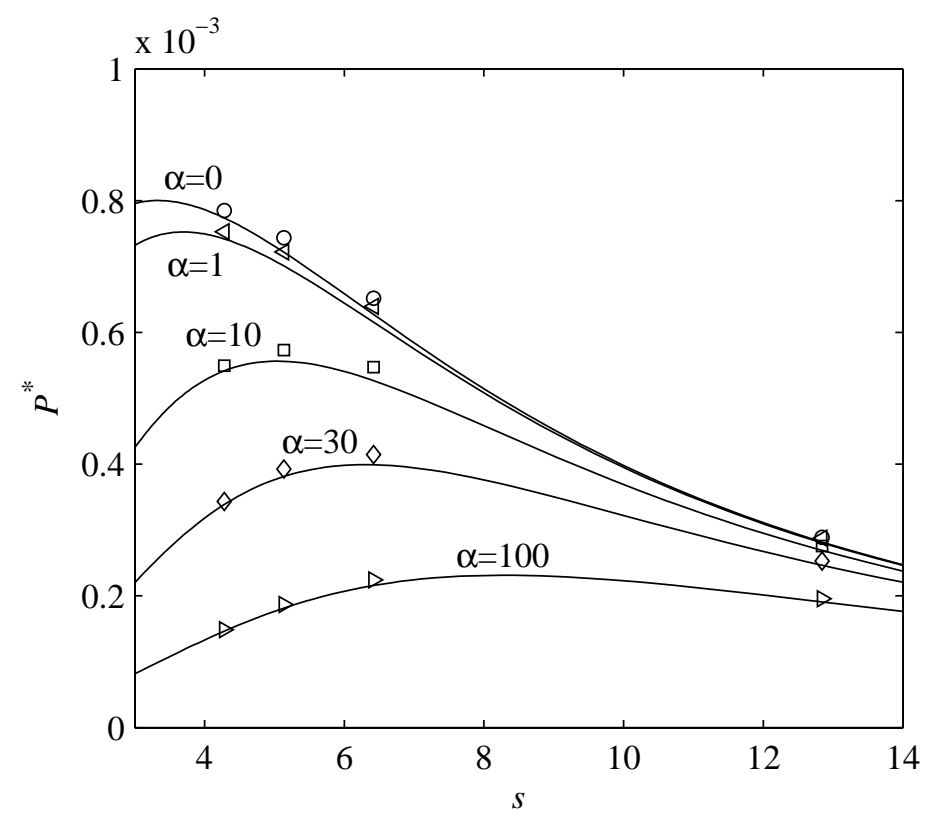

Figure 3: Normalized Power per unit cost for different values of $\alpha$ as function of the average turbine spacing $s$ (and $C_{T}^{\prime}=4 / 3$ ). Lines: obtained from model (Eqs. 18,13,15). Symbols: from large-eddy

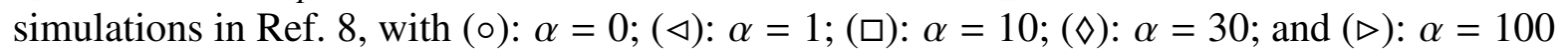

where $f\left(V(z)-V_{G}\right)$ corresponds to the driving Coriolis force in an Ekman layer, ${ }^{12}$ and $V(z)$ is the averaged span-wise velocity as function of the height. The left-hand side of Eq. (19) corresponds to $u_{* h i}^{2} \rho \ell_{x} \ell_{y}$, and also $\tau_{w}=\rho u_{* l o}^{2}$ (cf. previous subsection), such that

$$
u_{* h i}^{2}=u_{* l o}^{2}+\frac{1}{2} c_{f t}^{\prime} U_{d}^{2}
$$

Hence,

$$
\frac{U_{d}}{u_{* h i}}=\left(\frac{1-u_{* l o}^{2} / u_{* h i}^{2}}{c_{f t}^{\prime} / 2}\right)^{1 / 2} .
$$

This equation, in combination with Eqs. (13), and (15), are now used to express $U_{d} / u_{* h i}$ in Eq. (18). A combined easy-to-use analytical expression, directly expressing $U_{d} / G$ (cf. Eq. 18), is provided in the Appendix, relying on an approximate solution to Eq.(11).

\section{Optimal turbine spacing}

Based on the formulation for the normalized power $P^{*}$, we now make an evaluation of average windfarm power output as function of $C_{T}^{\prime}, \alpha$, and $s$. Moreover, the optimal average turbine spacing $s_{\text {opt }}$ is also investigated. As discussed in $\S 2.2$, we take $R o_{h}=2000$. Further, for the 'low' surface roughness we select $z_{0, l_{o}} / z_{h}=10^{-3}$ (cf. \$2.3). We first focus on situations where turbines are working in optimal operating conditions, with relative high values of $C_{T}^{\prime}$. This operation mode is encountered mainly in region II of a wind turbine's operation range. The effect of stalling or feathering turbines for constant power output at high wind velocities (region III of a wind turbine's operation range) on optimal turbine spacing will be briefly addressed at the end of the current section.

We first turn to the evaluation of the normalized power $P^{*}$. In Figure 3, $P^{*}$ (Eq. 18) is displayed as function of $s$ for different values of $\alpha$, and $C_{T}^{\prime}=4 / 3$. The normalized power is evaluated using the 
model proposed in the previous section, but next to that, results from the LES simulations of Calaf et $a l .{ }^{8}$ are also displayed. Large-eddy simulations were performed for different values of $s$, and $C_{T}^{\prime}$ : the average turbine power output $P$, and the induced surface roughness $z_{0, h i}$ are directly obtained from the simulations (cf. Refs. 8,9 for details on the simulation procedure, and set-up), and the respective values for $P^{*}$ are obtained using Eq. (16) and (11). It is clear from Figure 3 that the model for $P^{*}$ presented in the previous section, provides a reasonable fit of LES results at various average turbine spacings. Results in Figure 3 for $\alpha=0$ show that the turbine spacing which achieves maximum power output per acre is relatively small $\left(s_{\text {opt }} \approx 3\right)$. These low values (related to $\left.s<5\right)$ should be interpreted with care, as the LES simulations used to construct $P^{*}$ are not covering this region (cf. discussion at the beginning of Section 2.3). Moreover, when turbines are very closely spaced, turbulent fluctuations induced by the preceding row of turbines may start to reduce the effective power coefficient $C_{P}^{\prime}$ of the turbines, reducing the power converted to electricity (cf. Eq. 7). Further, at small distances other costs such as increased wake-turbulence induced fatigue damage and failures should be incorporated into the analysis. At higher values for $\alpha$, we find that the optimum shifts to higher values of $s$, for which our model for $P^{*}$ is better suited.

Before continuing an evaluation of the optimal turbine spacing as function of $\alpha$, the effect of turbine spacing on the power output per individual turbine is highlighted in Figure 4 for three values of $C_{T}^{\prime}$. In this figure, $P / P_{\infty}$ is displayed as function of $s$, where $P_{\infty}$ is the reference power output of a lone-standing turbine. It is appreciated that low values of $s(<10)$ significantly reduce the available power per turbine. This is a result of a lower available wind speed at hub height corresponding to a higher wind-farm induced surface roughness $z_{0, h i}$, and a slow-down of the ABL at constant geostrophic wind $G$. For instance, even at a relative large average spacing of $s \approx 10$, power output decreases by more then $20 \%$ compared to the power output of a lone-standing turbine. Obviously, the total farm power is also related to the number of turbines per acre (which is inversely proportional to $s^{2}$ ), such that optimal turbine spacings may be found at much lower values for $s$, as demonstrated in Figure 3.

In figure 4, we also added actual wind-farm data. To this end, we compared wind-speeds at the last row of turbines to those at the first row of turbines as obtained by the SCADA system for the Nysted and Horns Rev wind farms (reported in Frandsen et al 2007). ${ }^{17}$ In case of the Horns Rev wind-farm, $s_{x}=x_{y}=7$, and measurements were obtained at the $10^{\text {th }}$ row of turbines, $\pm 5 \mathrm{~km}$ downstream of the first row. For the Nysted wind farms, $s_{x}=10.5$, and $s_{y}=5.8$. Measurements were obtained at the $8^{\text {th }}$ row of turbines, $\pm 6 \mathrm{~km}$ downstream of the first row. Precise $C_{T}$ (or $C_{T}^{\prime}$ ) values are not reported. For both cases, the atmospheric boundary layer may not have reached the fully developed windturbine array limit, which we expect for wind-farms with horizontal extents exceeding 10-20 km. The measured data fall somewhat above the modeled normalized power in figure 4 but the agreement is reasonable given the various uncertainties involved in the analyses, measurements, and the lack of complete convergence towards a 'fully developed' limit.

The optimal turbine spacing $s_{\text {opt }}$ is now investigated as function of $\alpha$, and $C_{T}^{\prime}$. In the current work, it is not our intention to provide a detailed estimation of $\alpha$ based on current technical and economical parameters. Instead, we will investigate a broad range of possible values for this ratio. Nevertheless, it is useful to provide at least a rough idea of what a typical value of the parameter could be. Some representative numbers that can be used are motivated as follows. For lease of land, the average yearly payout per wind turbine nowadays is around $\$ 5,000$ for present typical spacings of $500 \mathrm{~m}$ by $500 \mathrm{~m}$ (see e.g., http://www.windustry.org/how-much-do-farmers-get-paidto-host-wind-turbines). So over a 20 year lease, this would be around $p_{L} \approx 0.4 \$ / \mathrm{m}^{2}$. In some regions, the purchase of the land may be an option. For instance in Texas one may estimate a cost of $\$ 1,000$ per acre (see e.g. http://recenter.tamu.edu/data/agp), or approximately $0.25 \$ / \mathrm{m}^{2}$, i.e., of similar order of magnitude to the cost of leasing. Representative cost of a wind-turbine can be found at http://www.windustry.org/how-much-do-wind-turbines-cost. The average cost is listed as $\$ 3.5 \times 10^{6}$ 


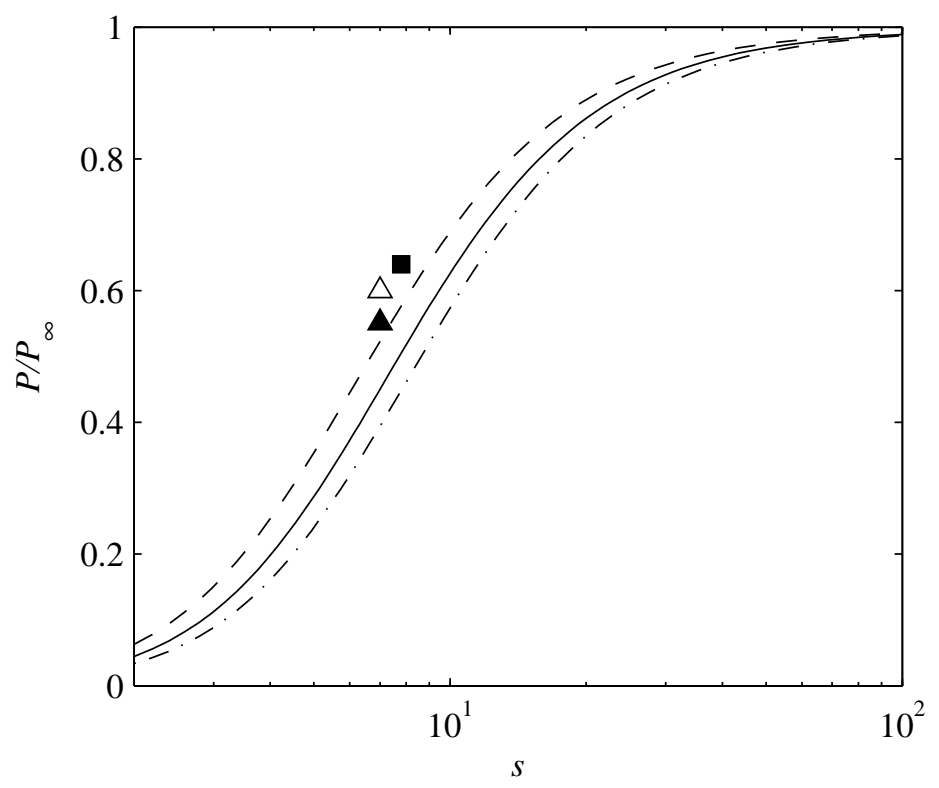

Figure 4: Power output per turbine as function of turbine spacing $s$, normalized with power output of a lone-standing turbine. (--) $C_{T}^{\prime}=1.0 ;(-) C_{T}^{\prime}=4 / 3 ;(-\cdot) C_{T}^{\prime}=5 / 3$. Symbols represent wind-farm measurements. $(\Delta, \mathbf{\Lambda})$ : Horns Rev wind farm $(s=7)$, last row compared to front row (west-east) at measured respectively 8.5 and $12.5 \mathrm{~m} / \mathrm{s}$ of wind speed (cf. Ref. 17, 18 ); ( $\square$ ): Nysted wind farm $\left(s^{2}=10.5 \times 5.8\right)$, last row compared to front row (west-east) measured with $8.5 \mathrm{~m} / \mathrm{s}$ of wind speed (cf. Ref. 17).

for a $2 \mathrm{MW}$ rated wind turbine. Using a representative turbine diameter of $70 \mathrm{~m}$ one arrives at a cost factor per square diameter of $\operatorname{cost}_{T} \approx 700 \$ / \mathrm{m}^{2}$. The corresponding parameter $\alpha$ is then roughly in the range of $1.5-3 \times 10^{3}$. In the current work, we will cover a range of values for $\alpha$ from $\alpha=0$ (corresponding to no costs per turbine) to $\alpha=10^{4}$.

In Figure 5 the optimal turbine spacing $s_{\text {opt }}$ is displayed as function of $\alpha$. By investigating various values of $\alpha$ and $C_{T}^{\prime}$, we found that only one global optimum exist for a given value of $\alpha$, and $C_{T}^{\prime}$ (see, e.g., Figure 3). The farm-power model provided in the previous section was implemented in MATLAB, and for the optimization, we employ the fminbnd function with bounds $0 \leq s \leq 40$. This method combines standard optimization algorithms, such as parabolic interpolation, and golden section search (cf., e.g, Ref. 19).

In Figure 5(a), the optimal spacing is given for three values of $C_{T}^{\prime}$. It is clear that $\alpha$ has a strong influence on $s_{\text {opt }}$, with optimal values ranging from $s \approx 4$ for $\alpha=1$ to $s \approx 25$ for $\alpha=10^{4}$. An analysis of the trends at large $\alpha$ suggests a scaling behavior of $s_{\text {opt }} \sim \alpha^{1 / 4}$, in the limit $\alpha \rightarrow \infty$. Obviously, for low values of $\alpha$ and low values of $s_{\text {opt }}$, other factors may play a decisive role in the selection of the average turbine spacing in wind farms (such as, e.g., constraints imposed by fatigue loading in closely placed turbines). For large values of $\alpha$ (e.g. $10^{3}<\alpha<10^{4}$, which may be economically more relevant), it is appreciated that optimal turbine spacing is larger than $s_{\text {opt }} \approx 15$. This is considerably larger than typical average spacings currently used in large wind farms both on and off shore (e.g., the well known Horns Rev wind farm off the coast of Denmark, has an average farm spacing of $s=7$ ).

In order to investigate the sensitivity of the optimal farm power output $P^{*}$ to variations of $s$ around the optimum $s_{\text {opt }}$, we evaluated sub-optimal solutions in Figure 5(b). To this end, we define the suboptimal spacing $s_{\eta}(\alpha)$ as the spacing that gives a power output of $P^{*}\left[s=s_{\eta}(\alpha)\right]=\eta P_{o p t}^{*}$ (and $\left.s<s_{\text {opt }}\right)$. Hence $s_{x}(\alpha)$ provides a spacing which is lower then $s_{\text {opt }}$, and has a power efficiency of 


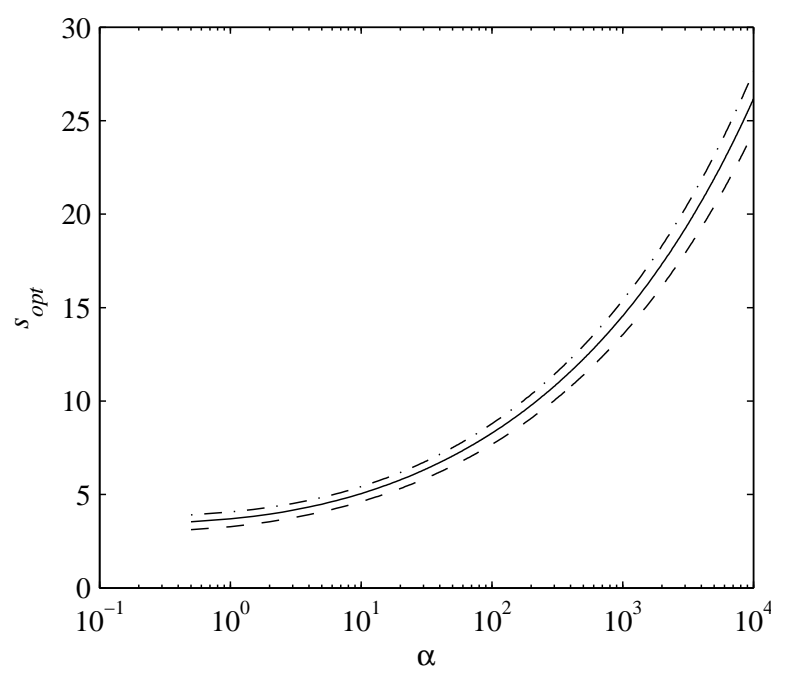

(a)

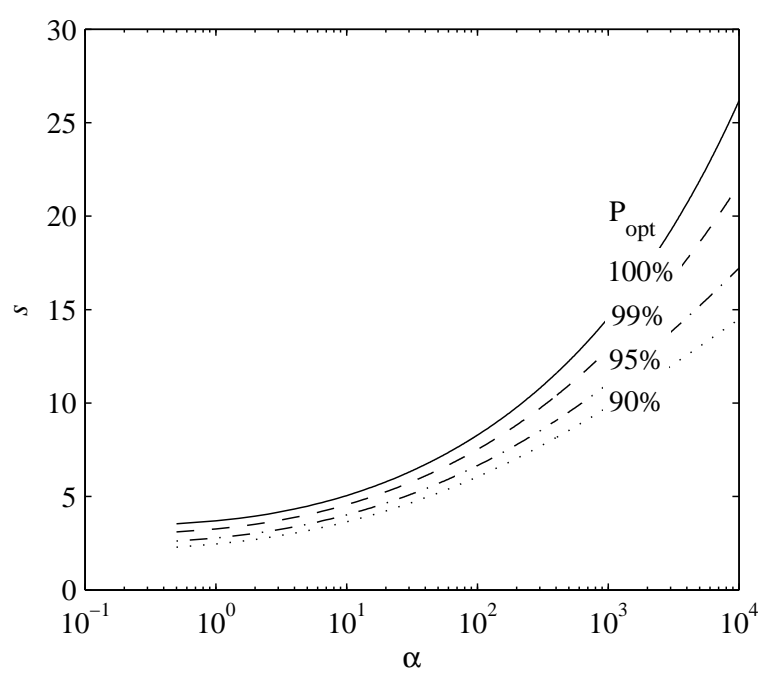

(b)

Figure 5: (a) Optimal turbine spacing $s_{\text {opt }}$ as function of the ratio $\alpha$ for different values of $C_{T}^{\prime}$ : (--) $C_{T}^{\prime}=1.0 ;(-) C_{T}^{\prime}=4 / 3$; and (-.) $C_{T}^{\prime}=5 / 3$. (b) Smallest turbine spacing as function of $\alpha$ (and for $\left.C_{T}^{\prime}=4 / 3\right)$ for which the normalized power output $P^{*}$ corresponds with $(-) 100 \% P_{o p t}^{*} ;(--) 99 \%$ of $P_{o p t}^{*} ;(-) 95 \$.$% of P_{o p t}^{*}$; and (..) $90 \%$ of $P_{o p t}^{*}$

$\eta$ compared to the power at optimal spacing. In Figure 5(b), results are shown for $s_{99 \%}, s_{95 \%}, s_{90 \%}$, together with $s_{\text {opt }}$. Especially at high values of $\alpha$, it is appreciated that a reduction in desired overall farm-ABL efficiency allows to reduce the spacing significantly. For instance, taking $\alpha=10^{4}$, and $\eta=95 \%$ allows to reduce the optimal spacing $s_{\text {opt }} \approx 25$ to a spacing of $s \approx 15$.

Finally we turn our attention to region III operation of wind turbines where turbine-power output is limited to a constant value, independent of wind speed, by pitching the turbine blades, either to feather or to stall the turbine. ${ }^{4}$ In the case of pitching to feather, the angle of attack of the turbine blades is decreased such that the lift forces decrease. In this case the thrust forces decrease, while, since the flow remains attached in this regime, the ratio $C_{P}^{\prime} / C_{T}^{\prime}$ remains constant. In the case of pitching to stall, the angle of attack of the turbine blades is increased, such that the turbine starts to stall. In this case, the ratio $C_{P}^{\prime} / C_{T}^{\prime}$ decreases, but the thrust force and the thrust coefficient $C_{T}^{\prime}$ does not decrease, and may initially even increase. Obviously, the different behavior of $C_{T}^{\prime}$ in both control methods, may differently affect the wind-farm ABL interaction.

In practice, wind-farm optimization of $P^{*}$ should be performed over the whole operating region of the turbine, weighted with statistical distribution of geostrophic wind speeds available at a certain location (e.g. assuming a classical Weibull distribution to characterize the wind-speed probability density function). ${ }^{10}$ In case power in region III operation is controlled by stalling the turbine, the optimal turbine spacing will not differ significantly from the results presented above for region II operation. In this case, $C_{P}^{\prime} / C_{T}^{\prime}$ is modulated by stalling the turbine blades, but $C_{T}^{\prime}$ remains roughly constant, close to its region II value. Hence, the control in region III does not affect the wind-farmABL interaction.

Region III operation becomes quite different when turbines are feathered to control the power output to a constant. In this case, $C_{P}^{\prime} / C_{T}^{\prime}$ is kept constant, and $C_{T}^{\prime}$ decreases by reducing the angle of attack of the turbine blades. As a result, the ABL partially speeds up. In Figure 6(a), this situation is demonstrated for different values of $\alpha$, and starting with a region-II value of $C_{T}^{\prime}=4 / 3$, and a turbine spacing $s_{\text {ref }}=s_{\text {opt }}$ which is optimally designed for region II operation. The figure shows the power output $P^{+}$normalized with the power output $P_{r e f}^{+}$at $C_{T}^{\prime}=4 / 3$. Especially for low values of $\alpha$ (for 


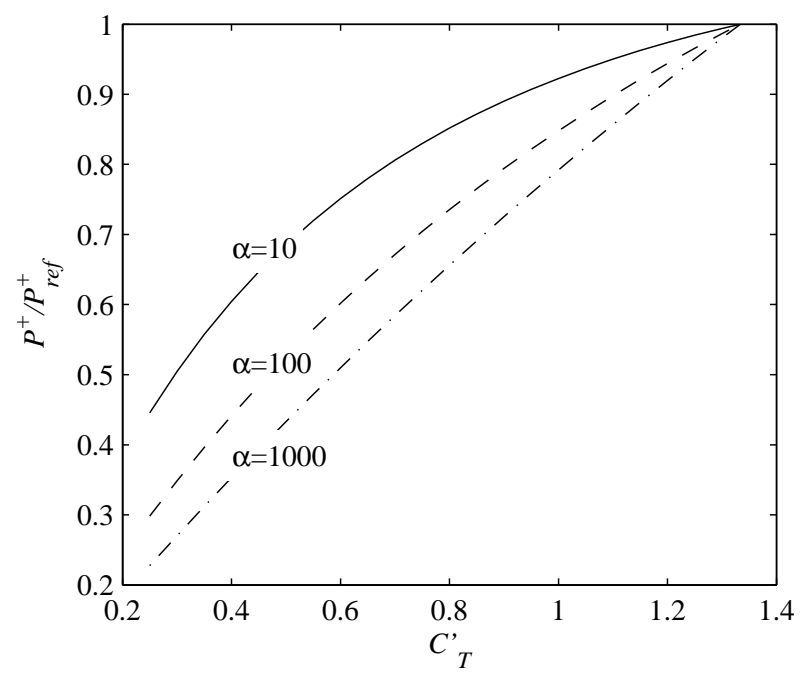

(a)

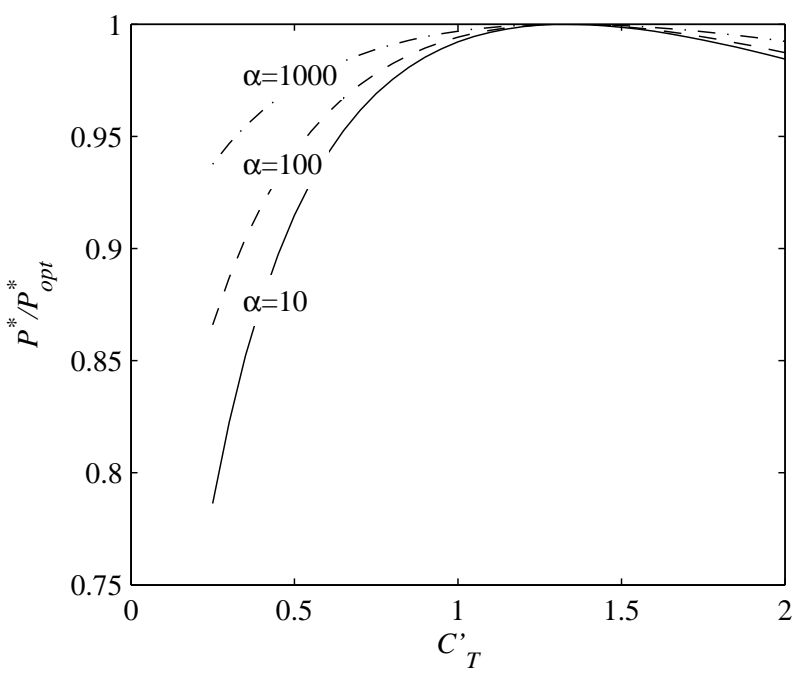

(b)

Figure 6: (a) Ratio of $P^{+}\left(s_{r e f}, C_{T}^{\prime}\right)$ to $P_{r e f}^{+}\left(s_{r e f}, 4 / 3\right)$ (with $s_{r e f}=s_{\text {opt }}\left(C_{T}^{\prime}=4 / 3\right)$, the optimal value for $\left.C_{T}^{\prime}=4 / 3\right)$ as function of the thrust coefficient $C_{T}^{\prime}$. (b) Dependence of $P^{*}\left(s_{\text {ref }}, C_{T}^{\prime}\right) / P_{\text {opt }}^{*}\left(s_{\text {opt }}, C_{T}^{\prime}\right)$ on $C_{T}^{\prime}$, with $s_{\text {ref }}=s_{\text {opt }}\left(C_{T}^{\prime}=4 / 3\right)$.

which $s_{\text {opt }}$ is low, cf. Figure 5) we find a large influence of the wind-farm-ABL interaction on the control. For lone-standing turbines, we would expect $P^{+}$to decrease linearly with $C_{T}^{\prime}$. Instead, for low values of $\alpha$, a decrease of $C_{T}^{\prime}$ leads to an acceleration of the ABL, such that $P^{+}$decreases less then linearly. For high values of $\alpha$ this effect diminishes, since here $s_{\text {opt }}$ is higher, approaching more and more the situation of lone-standing turbines.

Since feathering turbines in region III affects the ABL, this will also reflect on the optimal wind-turbine spacing in wind farms, which may differ from the optimal obtained for region-II operation (in contrast to stalling the turbines). To further illustrate this, Figure 6(b) shows the ratio $P^{*}\left(s_{\text {ref }}, C_{T}^{\prime}\right) / P_{\text {opt }}^{*}\left(s_{\text {opt }}, C_{T}^{\prime}\right)$ as function of $C_{T}^{\prime}$, where $P^{*}\left(s_{r e f}, C_{T}^{\prime}\right)$ is the normalized power output of a farm with spacing $s_{\text {ref }}$ designed to be optimal at an operational point with $C_{T}^{\prime}=4 / 3$, while $P_{o p t}^{*}\left(s_{\text {opt }}, C_{T}^{\prime}\right)$ is the normalized power output for a farm designed to be optimal for $C_{T}^{\prime}$. When $C_{T}^{\prime}$ is decreased (starting from $C_{T}^{\prime}=4 / 3$ ) it is appreciated that the ratio of $P^{*}$ to the optimal value decreases, as the turbine spacing $s_{\text {ref }}$ is not optimal for these lower values of $C_{T}^{\prime}$. Optimizing $P^{*}$ for the whole operation range of the turbines in a wind farm is then equivalent to optimization with on average lower $C_{T}^{\prime}$ values, leading to lower turbine spacings (cf. also Figure 5).

\section{CONCLUSIONS}

Following a recent computational study of very large wind farms, in which a new parametrization of effective roughness height was proposed, ${ }^{8}$ we explored in the current work implications on optimal spacing among wind turbines. The limit of "infinite wind farms", when the overlaying atmospheric boundary layer has become "fully developed", is relevant in practice for wind farms on flat terrain whose length exceeds the height of the atmospheric boundary layer by over an order of magnitude. Then the boundary layer has reached a new constant equilibrium height and turbulence levels no longer change with downstream direction. In this limit the power extraction is dominated by vertical entrainment of kinetic energy. ${ }^{8,15}$ For optimal wind turbine spacing, the figure of merit that has been used here is the total power extracted for a given geostrophic wind velocity. Depending on the ratio 
of land-surface costs and turbine costs, different optimal spacings have been obtained. For realistic cost ratios, we find that the optimal average turbine spacing may be considerably higher $(\sim 15 D)$ then conventionally used in current wind-farm implementations $(\sim 7 D)$.

Naturally, the conclusions reached here are subject to considerable limitations. The approach is based on parameterizations of wind-farm-ABL interactions under neutral stratification conditions, and assumes a flat terrain with no topography. Very often, for land-based wind farms the topography will locally affect the interactions and thus affect the optimal arrangement. For large offshore wind farms, the distribution of costs according to 'per-turbine' or 'per surface area' may be more difficult to specify and depend greatly on conditions of connectivity, typical sea states, distances to the coast, etc. It is also important to point out that the current findings are relevant to optimal spacing in the "fully developed wind turbine array boundary layer" for wind farms that are significantly larger than the fetch required for a surface disturbance to reach equilibrium with the entire ABL. Normally this is assumed to take about 10 times the height of the ABL, i.e. we may consider the present analysis to be relevant for wind farms larger than (say) $10 \mathrm{~km}$. For shorter wind farms, the optimal spacing may depend on location, as the front wind turbines will be operating under more powerful incoming winds.

Finally, the parametrization makes no distinction among span-wise and stream-wise spacings of wind turbines, or effects of staggering their locations (or considering a tilted inflow). As shown (e.g.) in LES, ${ }^{9}$ increases on the order of $5 \%$ can be expected in the extracted power when one staggers the turbines. The overall optimization trends as predicted here will vary slightly under such conditions, but we expect the major trends to be the same. Still, especially in locations with strong prevailing wind directions in which staggering can be an important part of the optimization, differences with present predictions may be expected. More accurate optimization and prediction of the optimal power for large wind farms (in which the detailed couplings with the ABL are crucial) will need to await more generally valid and accurate parameterizations of wind-turbine-ABL interactions. This should include effects of thermal stratification, wind turbine arrangements, and complex terrain.

\section{Acknowledgments}

The research of J.M. is supported by OPTEC (Optimization in Engineering Center - K.U.Leuven). The research of C.M. is supported by the National Science Foundation (grant \# CBET-0730922).

\section{APPENDIX}

In the current work, we rely partially on relations with an implicit formulation (e.g. Eq. 11), which we solve numerically by means of an iterative solver (cf. \$2.2). Here we present an easy-to-use alternative expression, which allows to evaluate $U_{d} / G$ (required to obtain the normalized power) directly, by using an approximation for Eq. (11). We begin by using an approximate fit to the solution of Eq. (11) similar to that used before: ${ }^{7,20}$

$$
\frac{G}{u_{* h i}} \approx \frac{1}{\kappa}\left(\ln R o-A_{*}\right)
$$

The parameter $A_{*}$ is a fitting parameter which depends on $R o$. For $10^{4}<R o<9 \times 10^{5}$, we find $A_{*} \approx$ 3.2, with a maximum relative error of $7 \%$ on the prediction of $G / u_{* h i}$ (this range for $R o$ corresponds with $R o_{h}=2000, C_{T}^{\prime}=4 / 3$, and $25>s>3$ encountered in the current work). 
Using the model expression for $z_{0, h i} / z_{h}$ (Eq. 15) in the approximation yields

$$
\frac{G}{u_{* h i}} \approx \frac{V_{w}}{\sqrt{1+c_{f t} V_{w}^{2} / 2}}+\frac{1}{\kappa}\left(\ln \left[R o_{h}\left(1+\frac{D}{2 z_{h}}\right)^{\frac{-v_{w}^{*}}{1+\gamma_{w}^{*}}}\right]-A_{*}\right)
$$

where $V_{w}$ is a dimensionless velocity given by the expression

$$
V_{w}=\frac{1}{\kappa} \ln \left[\frac{z_{h}}{z_{0, l o}}\left(1-\frac{D}{2 z_{h}}\right)^{\frac{v_{w}^{*}}{1+v_{w}^{*}}}\right]
$$

(the velocity $V_{w}$ may be understood as an extrapolation from below the turbine wake of the average wind-farm velocity at farm hub height). ${ }^{8}$ The wake viscosity $v_{w}^{*}$ is given by Eq. (14) $\left(v_{w}^{*} \approx 28 \sqrt{c_{f t} / 2}\right)$, $c_{f t}=\pi C_{T} / 4 s^{2}$ and $c_{f t} / c_{f t}^{\prime}$ depends upon the operating region (as described in the main text, for region II we use $\left.c_{f t} / c_{f t}^{\prime}=9 / 16\right)$. Starting from Eq. (21), the ratio of disk velocity to friction velocity, also needed in Eq. (18), can be simplified to read

$$
\frac{U_{d}}{u_{* h i}}=\frac{V_{w} \sqrt{c_{f t} / c_{f t}^{\prime}}}{\sqrt{1+c_{f t} V_{w}^{2} / 2}}
$$

Summarizing and further simplifying, the overall ratio $U_{d} / G$ required in Eq. (16) can be written as

$$
\frac{U_{d}}{G}=\sqrt{\frac{c_{f t}}{c_{f t}^{\prime}}}\left[1+\frac{\sqrt{1+\frac{c_{f t}}{2} V_{w}^{2}}}{\kappa V_{w}}\left(\ln \left[R o_{h}\left(1+\frac{D}{2 z_{h}}\right)^{\frac{-v_{w}^{*}}{1+v_{w}^{*}}}\right]-A_{*}\right)\right]^{-1}
$$

Equation (26) provides an estimate of $U_{d} / G$ with a maximum relative error of $7 \%$ due to the approximation in Eq. (22) (using $A_{*}=3.2$ ). The expression should be handled with care when evaluating the normalized farm power (Eqs. 16,18), which depends on $\left(U_{d} / G\right)^{3}$. Using the approximate fit of Eq. (26), this yields a maximum relative error of about $20 \%$. This is the reason that in the current work, we selected not to use the approximation in Eq. (22), as discussed above (see also \$2.2). Nevertheless, since relative trends will be reasonably well predicted it is still useful to have such simplified expressions available for more qualitative parameter explorations.

\section{References}

1. US Department of Energy. 20\% wind energy by 2030: Increasing wind energy's contribution to U.S. electricity supply, 2008.

2. Commission of the European Communities. A European strategic energy technology plan technology map, 2007.

3. Snel H. Review of the present status of rotor aerodynamics. Wind Energy 1998; 1 : 46-49.

4. Burton T, Sharpe D, Jenkins N, Bossanyi E. Wind Energy Handbook. John Wiley \& Sons: New York, 2001.

5. Vermeer LJ, Sorensen JN, Crespo A. Wind turbine wake aerodynamics. Progress in Aerospace Sciences 2003; 39 : 467-510. 
6. Frandsen S. On the wind speed reduction in the center of large clusters of wind turbines. $J$. Wind Eng Indust. Aerodyn. 1992; 39 : 251-265.

7. Frandsen S, Barthelmie R, Pryor S, Rathmann O, Larsen S, Højstrup J, Thøgersen M. Analytical modelling of wind speed deficit in large offshore wind farms. Wind Energy 2006; 9 : 39-53.

8. Calaf M, Meneveau C, Meyers J. Large eddy simulation study of fully developed wind-turbine array boundary layers. Physics of Fluids 2010; 22 : Art no 015110. DOI:10.1063/1.3291077

9. Meyers J, Meneveau C. Large eddy simulations of large wind-turbine arrays in the atmospheric boundary layer. In 48th AIAA Aerospace Sciences Meeting Including the New Horizons Forum and Aerospace Exposition, January 2010. Art no AIAA2010-827.

10. Manwell JF, McGowan JG, Rogers AL Wind Energy Explained: Theory, Design and Application Wiley, 2002.

11. Jimenez A, Crespo A, Migoya E, Garcia J Advances in large-eddy simulation of a wind turbine wake. J. of Physics: Conference Series, 2007, 75 : 012041.

12. Tennekes H, Lumley JL. A first Course in Turbulence. The MIT Press, 1972.

13. Castro IP. Rough-wall boundary layers: mean flow universality. Journal of Fluid Mechanics 2007; 585 : 469-284.

14. Wu Y-T, Porté-Agel F. Large-Eddy Simulation of Wind-Turbine Wakes: Evaluation of Turbine Parameterizations. Boundary-Layer Meteorology, DOI 10.1007/s10546-010-9569-x, published online December 2010.

15. Cal RB, Lebrón J, Castillo L, Kang HS, Meneveau C. Experimental study of the horizontally averaged flow structure in a model wind-turbine array boundary layer. J. Renewable Sustainable Energy 2010; 2 : Art no 013106. DOI:10.1063/1.3289735

16. Fuglsang P, Thomsen K. Cost optimization of wind turbines for large-scale off-shore wind farms. Technical Report Ris $\varnothing-R-1000(E N)$, Ris $\varnothing$ National Laboratory, Roskilde, Denmark, 1998.

17. Frandsen S, Barthelmie R, Rathmann O, Jørgensen HE, Badger J, Hansen K, Ott S, Rethore P-E, Larsen SE, Jensen LE The shadow effect of large wind farms: measurements, data analysis and modelling. Technical Report Ris $\varnothing-\mathrm{R}-1615$, Ris $\varnothing$ National Laboratory, Roskilde, Denmark, 2007

18. Barthelmie RJ, Rathmann O, Frandsen ST, Hansen K, Politis E, Prospathopoulos J, Rados K, Cabezón D, Schlez W, Phillips J, Neubert A, Schepers JG, van der Pijl SP Modelling and measurements of wakes in large wind farms Journal of Physics: Conference Series 2007; 75: Art no 012049 .

19. Brent RP. Algorithms for Minimization without Derivatives. Prentice-Hall: Englewood Cliffs, New Jersey, 1973.

20. Emeis S, Frandsen S. Reduction of horizontal wind speed in a boundary layer with obstacles. Boundary-Layer Meteorology 1993; 64 : 297-305. 